täts- und Koordinationsfunktion übernehmen. Sie sind auf keinen Fall als nationalstaatliche, formale Kontrolleure aufzubauen, sondern vielmehr im Geiste von «neutralen» Dienstleistern für die Klubs und Erschaffern von kreativen Kooperationsmilieus.

\section{Literatur}

FREY, R. L. (1985): Regionalpolitik: eine Evaluation. Publikation des Schweizerischen Nationalfonds aus den Nationalen Forschungsprogrammen, Bd. 34,6, Bern.

HAEFLIGER, Ch. J. (1995): Sechsmal die "Außen-Schweiz": Die Kantone und INTERREG II. Vortrag gehalten an der Tagung "Aufbruch der Kantone" am 27. Oktober 1995 in Basel (Vortragsmanuskript).
LEZZI, M. (1994): Raumordnungspolitik in europäischen Grenzregionen zwischen Konkurrenz und Zusammenarbeit. Untersuchungen an der EG-Außengrenze Deutschland/ Schweiz. Wirtschaftsgeographie und Raumplanung, Vol. 20, Zürich (Diss. geogr. Zürich).

LEZZI, M. (1993): Raumordnungspolitik in den 90er Jahren zwischen Regionalismus und Supranationalisierung? Untersuchung in deutschen und schweizerischen Grenzregionen. In: 49. Deutscher Geographentag Bochum 1993 hrsg.v. D. BARSCH und H. KARRASCH, Bd. 4, S. 66-74. Stuttgart.

ROREP (1995): Deklaration zum Zukunftsraum Schweiz. hrsg. v. Schweizerischen Studiengesellschaft für Raumordnungs- und Regionalpolitik (ROREP).

STRAUBHAAR, T. (1993): Zur optimalen Größe eines integrierten Wirtschaftsraumes. Das Konzept des funktionalen Föderalismus. Diskussionsbeiträge zur Wirtschaftspolitik, hrsg. v. Institut für Wirtschaftspolitik der Universität der Bundeswehr Hamburg.

\title{
Bevölkerung und Bevölkerungsentwicklung
}

\section{Einleitung}

Bevölkerungsverteilung und Bevölkerungsdynamik werden vielfach als Schlüsselgrößen zur Beurteilung der wirtschaftlichen Stärke der Teilräume eines Landes verwendet, da demographisches und ökonomisches Wachstum in der Vergangenheit empirisch oft hoch korreliert waren. In den Raumwissenschaften wird allerdings mehr Gewicht auf Strukturmerkmale der Bevölkerung und die Komponenten des Bevölkerungswachstums - d.h. auf den Geburtenüberschuß und die Wanderungen - gelegt. Als Ausgangspunkt des knappen Überblicks zur Bevölkerungsverteilung und Bevölkerungsentwicklung in der Schweiz wird ein spezieller und kompliziert hergeleiteter Indikator beigezogen: Es handelt sich um die Saldi der Binnenwanderungen nach den sieben Fünfjahresperioden' von 1960 bis 1995, mithin der Phase, in der in der
Schweiz eine aktive räumliche Ausgleichspolitik betrieben worden ist. Als geographische Bezugsräume werden sieben Regionstypen verwendet. Die Darstellung dieses Indikators über den Beobachtungszeitraum zeigt eine kontinuierliche Ausgleichstendenz der Wanderbewegungen zwischen den Regionstypen, seit Beginn der 80er Jahre gar eine eigentliche Trendumkehr.

Martin Schuler, dipl. Geogr., Institut de Recherche sur l'Environnement Construit (IREC), EPFL, 14, avenue de l'EgliseAnglaise, 1001 Lausanne 
$\%$

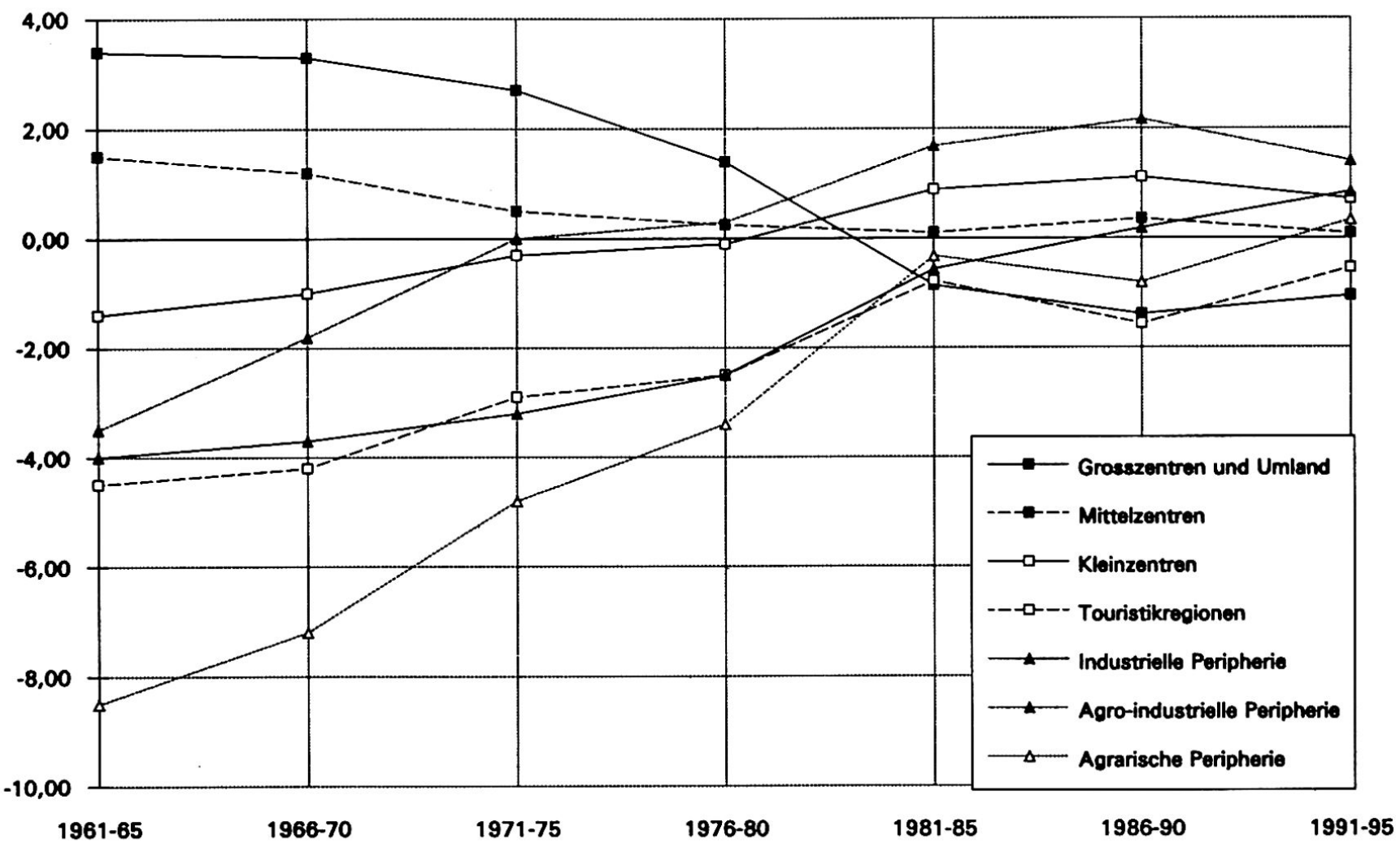

Grafik 1

Die verwendete Regionstypologie unterscheidet nach einer abgestuften Zentralitätshierarchie sowie nach der Wirtschaftsstruktur. Sie ist als Zusammenzug der 106 MS-Regionen anhand der Volkszählungsdaten 1980 bestimmt worden. Weder der konzeptionelle Ansatz der Typologie noch die Zuteilung der einzelnen Teilräume zu den Regionstypen brauchen für die gesamte Zeitspanne aussagekräftig zu sein. Die Darstellung kann im geschichtlichen Rückblick deshalb doppelt gelesen werden, nämlich entweder als Ausdruck veränderter Wanderungsbewegungen in einem gegebenen Raum oder als Effekt räumlicher Maßstabsverschiebungen im Laufe der Zeit.

Während der 60er und 70er Jahre blieben großzentrale Regionen und ihre Umlandgebiete die Hauptgewinner der Binnenwanderungen, gefolgt von den mittelzentralen Regionen. Nur diese beiden Regionstypen konnten positive Wanderungssaldi verzeichnen, während die übrigen fünf Raumeinheiten Einwohnerverluste durch die interne Migration erlitten. Diese Verluste fielen in kleinzentralen Regionen schwach aus, in agroindustriellen, industriellen und touristischen Regionstypen mittelstark und in agrarischen Regionen, d. h. in räumlich zumeist in den Alpen und Voralpen gelegenen Gebieten, stark. Die Saldi nahmen im Verlaufe der ersten vier Perioden in allen Regionstypen regelmäßig ab und betrugen um 1975-80 nur noch knapp die Hälfte der Werte von 1960-65. So sanken die Gewinne der Großzentren von 3,4\% auf 1,4\%; während sich die Verluste im agrarischen Regionstyp von $-8,5 \%$ auf $-3,4 \%$ reduzierten. Es ist auffällig, daß sich zwischen den sieben Regionstypen kaum Rangverschiebungen ergaben.

In der ersten Hälfte der 80er Jahre kam es zur eigentlichen Umkehr bei den Wanderungsbewegungen. Die Großzentren und ihr Umland wurden zu Migrationsverlierern; Kleinzentren und agroindustrielle Regionen (beide überwiegend im Zwischenraum des Mittellandes gelegen) wurden zu den stärksten Gewinnern, während die periphersten Regionstypen ins Mittelfeld aufrückten und nun annähernd ausgeglichene Bilanzen aufwiesen. Nach einem leichten Öffnen der Schere in der zweiten Hälfte der 80er Jahre haben sich seit 1990 die Wanderungssaldi wiederum angeglichen. Migrationsverlierer sind heute die Regionen der großen und mittleren Zentren; alle anderen Regionstypen verzeichnen Gewinne. 
In der zeitlichen Entwicklung der Binnenwanderungssaldi nach den ausgewählten Raumniveaus zeigen sich erstaunlicherweise kaum Abhängigkeiten von der jeweiligen konjunkturellen Situation; weder die wirtschaftliche Rezession 1974-76 noch der schwache Konjunkturrückgang zu Beginn der 80er Jahre und auch nicht der Einbruch ab 1991 haben die generelle Tendenz modifiziert.

Als Erklärungsversuche der langjährigen, ungebrochenen Tendenz zum Ausgleich der Binnenwanderungen können andere Migrations- und Mobilitätsformen beigezogen werden, die Entwicklung der Mobilitätsintensität oder auch feinere Aufschlüsselungen nach demographischen und sozialen Merkmalen der Migranten:

- Die Zuzüge aus dem Ausland waren in der betrachteten Zeitspanne für die wirtschaftliche Entwicklung und die räumliche Bevölkerungsverteilung ebenso wichtig wie die Binnenwanderungen. In großstädtischen und touristischen Gebieten sind in den meisten der betrachteten Perioden mehr Zuwanderer aus dem Ausland gezählt worden als aus den übrigen Regionen der Schweiz; die Intensität der internationalen Wanderungen und deren Saldi zeigen eine starke Abhängigkeit vom Wirtschaftsverlauf.Zudem sind die Binnenwanderungen der Ausländer innerhalb der Schweiz vielfach gegenläufig zu denen der Einheimischen verlaufen, da die Ausländer aus arbeitsmarktpolitischen Gründen bei ihrer ersten Einreise in schwache Wirtschaftszweige, schlecht bezahlte Anstellungen und tendenziell auch in periphere Regionen gelenkt wurden. Die Folgewanderungen der zumeist jungen Immigranten sind Ausdruck ökonomischer und räumlicher "Aufwärtsbewegungen».

- Die Binnenwanderungsbewegungen der Schweizer haben sich zwar in der Bilanz räumlich angeglichen, doch ist ihr Umfang über weite Distanzen ganz erheblich zurückgegangen. Die Wahrscheinlichkeit, innerhalb von fünf Jahren zu wandern, hat sich nach den verschiedenen Raumebenen wie folgt entwickelt: here Kohorten. Die Wanderungsbewegungen der Jungen zielen nach wie vor eher auf die Zentren, doch der Anteil junger Wegzüger aus peripheren Gebieten ist deutlich rückläufig. Zudem ist die früher viel stärkere Zentrenorientierung der Wanderbewegungen der jungen Frauen abgeklungen.

- Längere Ausbildungszeiten und vermehrt dezentral lokalisierte Bildungseinrichtungen haben den Wegzug der Jungen aus dem Elternhaus hinausgeschoben und damit einen Teil der früher üblichen Folgewanderungen verzögert oder substituiert. Zusammen mit der Tatsache, daß die meisten Jugendlichen heute bereits in groß- oder mittelzentralen Regionen aufwachsen, bedeutet dies, da $\beta$ viele unter ihnen gar nie die Region wechseln (müssen). Für die Angehörigen der mittleren Generation erschwert die doppelte Erwerbstätigkeit Regionswechsel. Schließlich weisen verschiedene Indizien darauf hin, daß sich ein Wandel in der Beurteilung der regionalen Disparitäten ergeben hat. Zwar sind die objektiven wirtschaftlichen Unterschiede - etwa im Lohnniveau - kaum geringer geworden, doch hat sich bis nach 1990 deren absolutes $\mathrm{Ni}$ veau erhöht. In den räumlichen Präferenzen haben Aspekte der Lebensqualität eine stärkere Berücksichtigung finden können. Das entscheidende Element in der Umorientierung der Wanderungsbewegungen ist jedoch in einer substitutiven Mobilitätsform zu suchen, dem vermehrten Arbeits-(und Ausbildungs-)Pendeln.

Der Anteil der Erwerbstätigen, die außerhalb der Wohngemeinde arbeiten, hat seit 1960 jährlich um ein Prozent zugenommen, die zurückgelegte Distanz im Jahresmittel um 80 Meter, der Zeitaufwand dagegen nur um 10 Sekunden. Dank verbesserten Erreichbarkeitsverhältnissen und der Wahl schnellerer Verkehrsmittel haben sich die Verkehrsströme intensiviert, wobei die Pendlerdistanzen in der Schweiz im internationalen Vergleich noch immer relativ kurz erscheinen.

Tabelle 1 Anteil Binnenwanderungszuzüge in Prozenten, nach Raumeinheiten (Vergleich über drei Fünfjahresperioden)

\begin{tabular}{|l|l|l|l|l|}
\hline $\begin{array}{l}\text { Raumeinheit } \\
\text { (Anzahl Einheiten) }\end{array}$ & $1965-70$ & $1975-80$ & $1985-90$ & Rückgang \\
Gemeinde (3021) & 19,0 & 18,9 & 18,2 & $65-70$ bis $85-90$ \\
Kleinregion (106) & 12,9 & 11,6 & 10,6 & $-4 \%$ \\
Kanton (26) & 8,3 & 7,0 & 6,2 & $-25 \%$ \\
Großregion (16) & 7,5 & 6,1 & 5,3 & $-29 \%$ \\
Sprachregion (4) & 1,9 & 1,4 & 1,1 & $-40 \%$ \\
\hline
\end{tabular}

- Die Struktur der Migranten hat sich im Zeitablauf nicht grundsätzlich gewandelt; junge Erwachsene stellen nach wie vor die mobilste Bevölkerungsgruppe dar, doch ist die Mobilität der 15-19jährigen deutlich gesunken, während die 25-29jährigen heute leicht mobiler sind als frü-
Die Pendlerziele sind zwar räumlich diffuser geworden (und damit für die Benutzer öffentlicher Verkehrsmittel unattraktiver), doch läßt sich eine verstärkte Konzentration der Arbeitsplätze auf großzentrale Regionen und innerhalb derselben vorzugsweise auf das weitere Umland 
beobachten. Das relative Arbeitsplatzwachstum fiel in den durch den Nationalstraßenbau neu erschlossenen Zwischenachsen im Mittelland am stärksten aus. Damit hat die Arbeitsplatzentwicklung die Ausgleichstendenz der Bevölkerung nicht oder nur zum kleinen Teil nachvollzogen. Analog zu den früheren Kettenwanderungen von peripheren zu kleinzentralen, zu mittel- und großzentralen Regionen findet sich heute ein Muster von Kettenpendelbewegungen: Die Klein- und Mittelzentren strahlen weit in ihr Hinterland aus, sind aber ihrerseits an die Großzentren tributär.

Die Analyse der Pendlerbewegungen relativiert das Bild zunehmend ausgeglichener Raumstrukturen und stützt eher die Hypothese der räumlichen Maßstabsverschiebung als eines Übergangs von klein- zu großregionalen Strukturen.

Das Gebiet, dem in der Schweiz ein eigentlicher Peripheriecharakter zugesprochen werden kann, hat sich dauernd verkleinert. Während noch in den 60 er Jahren die meisten Bergregionen und ländlichen Gebiete wirtschaftlich wie demographisch rückläufig gewesen waren, brachten die 70er Jahren einen Einbruch in den industriell geprägten Räumen mit besonders starkem Akzent im Jura. In den 80er Jahren war die Bevölkerungsentwicklung dagegen nur mehr in wenigen Peripherregionen rückläufig, so im Gotthardgebiet (Uri, Leventina, Surselva, Oberhasli) sowie in einzelnen abgelegenen Gemeinden im Jura, im Entlebuch und Emmental und einigen Alpentälern. Diese Tendenz hält nun auch noch nach 1990 an.

\section{Anmerkungen}

' Die Berechnungen sind methodisch nicht ganz vergleichbar, denn die sieben Fünfjahresperioden mußten nach drei unterschiedlichen Verfahren gemessen werden:

a) Die Angaben für 1966-70 und 1976-80 beruhen auf den Volkszählungsergebnissen von 1970 und 1980. Die Frage nach dem "Wohnort vor fünf Jahren" erlaubte das Berechnen von Matrizen der Binnenwanderungen. Die Migrationen werden in der Volkszählung nach dem wirtschaftlichen Wohnsitzbegriff erfaßt.

b) Für die beiden Fünfjahresperioden 1961-65 und 1971-75 existiert keine Migrationsstatistik. In einem indirekten Verfahren sind die Wanderungssaldi geschätzt worden als Differenz zwischen der Gesamtentwicklung der Bevölkerung zwischen zwei Volkszählungsstichdaten einerseits und andererseits den Binnenwanderungssaldi der zweiten Jahrzehnthälfte gemäß der Volkszählung sowie der natürlichen Bevölkerungsentwicklung und den internationalen Wanderungen (die ihrerseits aus der Bestandesänderung der Ausländer unter Berücksichtigung der Geburten, Todesfälle und Einbürgerungen hervorgehen).

c) Die Angaben für die Perioden 1981-85, 1986-90 und 1991-95 stammen aus der BFS-Statistik ESPOP, die jährlich gemeindeweise $\mathrm{Zu}$ - und Wegzüge nach drei institutionellen Ebenen (innerkantonal, interkantonal und international) erhebt. Im Gegensatz zu den Daten aus den Volkszählungen werden in dieser Statistik die Wandernden nach dem zivilrechtlichen Wohnsitzbegriff erfaßt. Die Periode 1991-95 entspricht den auf fünf Jahre hochgerechneten Resultaten der Jahre 1991-94. 